\title{
CAPTURING FINE DETAILS INVOLVING LOW-COST SENSORS - A COMPARATIVE STUDY
}

\author{
N. Rehany*, A. Barsi, T. Lovas \\ Department of Photogrammetry and Geoinformatics, Budapest University of Technology and Economics, Budapest, Hungary \\ (rehany.nikolett, barsi.arpad, lovas.tamas)@epito.bme.hu
}

\section{Commission II}

KEY WORDS: Laser scanning, object scanning, amateur sensors, low-cost sensors, comparison

\begin{abstract}
:
Capturing the fine details on the surface of small objects is a real challenge to many conventional surveying methods. Our paper discusses the investigation of several data acquisition technologies, such as arm scanner, structured light scanner, terrestrial laser scanner, object linescanner, DSLR camera, and mobile phone camera. A palm-sized embossed sculpture reproduction was used as a test object; it has been surveyed by all the instruments. The result point clouds and meshes were then analyzed, using the arm scanner's dataset as reference. In addition to general statistics, the results have been evaluated based both on 3D deviation maps and 2D deviation graphs; the latter allows even more accurate analysis of the characteristics of the different data acquisition approaches. Additionally, own-developed local minimum maps were created that nicely visualize the potential level of detail provided by the applied technologies. Besides the usual geometric assessment, the paper discusses the different resource needs (cost, time, expertise) of the discussed techniques. Our results proved that even amateur sensors operated by amateur users can provide high quality datasets that enable engineering analysis. Based on the results, the paper contains an outlook to potential future investigations in this field.
\end{abstract}

\section{INTRODUCTION}

Nowadays many surveying technologies are available to produce the point cloud of an object; short range object scanners, medium and long range laser scanners, methods based on photogrammetry and other techniques. There are significant differences regarding the performance of the particular devices: resolution, accuracy, measurement speed and, of course, the price. The different technologies are usually used for different purposes but it does not mean necessarily that a technology is suitable for measuring only a particular type of object. Producing the most accurate and detailed point cloud or 3D modell is not always the primary goal; the most appropriate technology is to be chosen instead. That means that besides the expected resolution, point density, accuracy, the time, expertise, costs and other resource needs are to be considered. Obviously all technologies have their own barriers, therefore a complex optimization process has to be solved.

Does the accuracy increase along with the costs? Do low-cost sensors produce low quality data? What are the software requirements of the particular devices and how long does the data processing take and what level of expertise does it require? Further sections of the paper aim at providing the answers for such questions by investigating how different point cloud producing technologies are suitable for surveying fine details of small objects. The 3D model of the test object is produced by different technologies then deviations from reference model are analyzed.

\section{SURVEYING}

The subject of surveying, our test object is a small, $12.5 \mathrm{~cm} \times 9 \mathrm{~cm}$ $\mathrm{x} 3.5 \mathrm{~cm}$, lacquered, gypsum sculpture reproduction with Maya

* Corresponding author figure, see Figure 1 . The relief bulges only few millimeters from the upper plane of the object and small cracks can be found on the surface that are not deeper and wider than a millimeter. Since the surface of the object is fragmented and contains lot of cracks, it seems to be appropriate test object for a comparative study.

Our goal was to investigate how the point clouds and 3D models of the object provided by the different surveying technologies represent the real object surface. We intended to involve both professional, top-of-the-line instruments and devices used in our everyday life. For sure, our list could have been extended by several other devices.

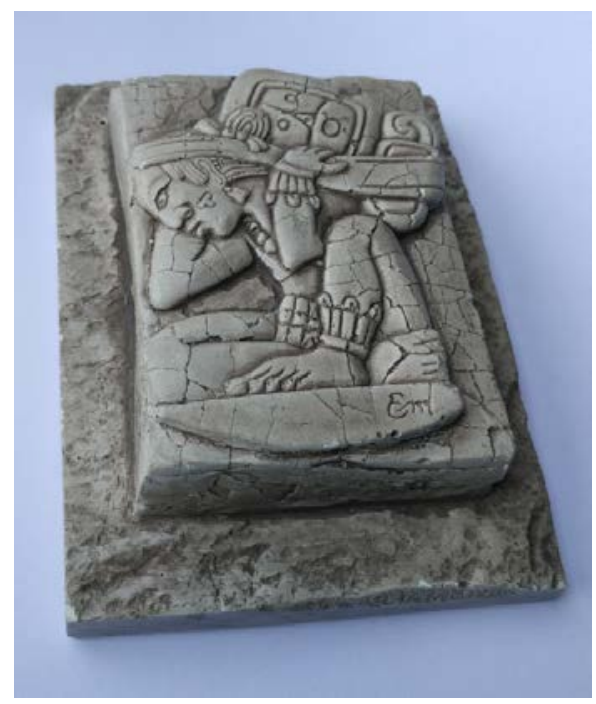

Figure 1. The test object 


\begin{tabular}{|c|c|c|c|c|c|c|}
\hline & Accuracy $^{1}$ & Resolution $^{1}$ & Capturing speed ${ }^{1}$ & $\begin{array}{l}\text { Measurement } \\
\text { time }\end{array}$ & $\begin{array}{l}\text { Approximate } \\
\text { price }^{2}\end{array}$ & Portability \\
\hline Artec & $0.1 \mathrm{~mm}$ & $0.5 \mathrm{~mm}$ & 2 million points/s & 5 minutes & 14000 EUR & moderate \\
\hline TLS & $\pm 2 \mathrm{~mm}$ & $1.5 \mathrm{~mm}$ at $10 \mathrm{~m}$ & 976000 points/s & 10 minutes & 30000 EUR & poor \\
\hline ScanArm & $0.076 \mathrm{~mm} / \mathrm{inch}$ & N/A & 45120 points/s & 10 minutes & 77000 EUR & poor \\
\hline Camera & N/A & $6000 \times 4000$ & N/A & 5 minutes & 600 EUR & good \\
\hline Mobile & N/A & $4208 \times 2368$ & N/A & 5 minutes & 300 EUR & good \\
\hline Sense & N/A & $\begin{array}{r}\mathrm{X} / \mathrm{Y} 0.9 \mathrm{~mm} \\
\mathrm{Z} 1 \mathrm{~mm}\end{array}$ & $30 \mathrm{fps}$ & 5 minutes & 400 EUR & moderate \\
\hline
\end{tabular}

Table 1. Technical specifications of the devices

The following instruments have been involved; Table 1 contains their technical specification:

- $\quad$ Artec Eva structured light scanner,

- $\quad$ Faro focus 3D S120 terrestrial laser scanner,

- $\quad$ Faro Edge ScanArm with Laser Line Probe measurement head,

- $\quad$ Canon EOS 760D camera with Canon Zoom Lens EF 28105mm 1:3.5-4.5 USM,

- Xiaomi Mi 4i (13MP Sony/Samsung camera, f/2.0) mobile phone,

- $\quad 3 D$ Sytems Sense 3D scanner.

Structured light scanners (SLS) project a pattern onto the object and capture photos then calculate the 3D coordinates of the surface points based on the reflected pattern (Fofi et al., 2004, Moons et al., 2008). Some scanners use visible (e.g. Artec) while others use invisible, infrared (e.g. Sense) light patterns. The already measured and matched parts of the model are visible during scanning in both cases so the model can be supplemented immediately onsite with the missing, not measured parts, i.e. the holes can be filled.

According to the manufacturer's recommendation, the Artec scanner is appropriate for measuring middle-size objects. It is mostly applied for industrial design and manufacturing (reverse engineering, quality control, rapid prototyping, aerospace), healthcare (orthopedics, prosthetics, plastic surgery, custom wheelchairs), art and design (heritage preservation, architecture, fashion), science and education purposes. The scanner has $0.1 \mathrm{~mm}$ measurement accuracy and is capable of producing textured surface model. Since Artec Eva must be connected to a notebook or computer during scanning, it is not fully portable and compact.

Sense scanner is to be used for scanning different sized objects in range from $0.2 \times 0.2 \times 0.2 \mathrm{~m}$ to $2 \times 2 \times 2 \mathrm{~m}$ (e.g. heads and bodies of people). Since our test object is smaller, additional objects were placed around it to support the measurement. This scanner also must be connected to a computer or laptop so it is not fully portable. However, its low price makes it affordable for the wide public.

The potential of using amateur cameras to produce point clouds has been also investigated. The principle of pixel-based 3D reconstruction is that the software finds the corresponding image points between overlapping images, then calculates the position and orientation of capturing and determines the 3D coordinates of the object's points based on spatial triangulation (Moons et al., 2008, Furukawa and Hernandez, 2015). The technique enables

1 From technical specifications provided by manufacturers

2 Prices from suppliers' websites matching color information to the points. There are many available software using different algorithms (Wikipedia, 2017), which are either accessible for free or has commercial licence; the latter usually has trial version. For the test, images have been captured using automatic settings by both DSLR camera (66 images) and mobile phone camera (65 images). Figure 2 shows the image locations and orientations in both cases; we intended to take reasonable number of images from positions that enable 3D reconstruction. Obviously, imaging geometry has significant effect on modelling accuracy (Moons et al., 2008, Furukawa and
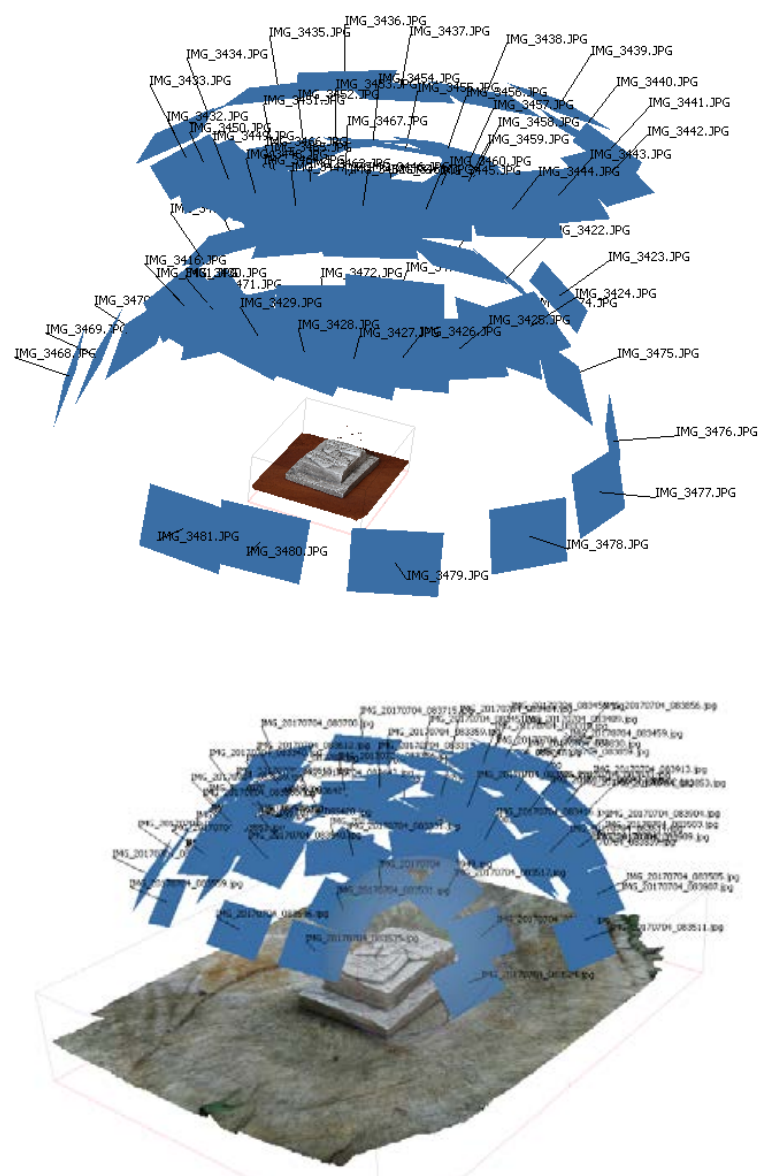

Figure 2. Position and orientation of the captured images in case of camera (up) and mobile phone (down) 


\begin{tabular}{|l|c|r|r|r|c|c|c|}
\cline { 2 - 8 } \multicolumn{1}{c|}{} & $\begin{array}{c}\text { Point density } \\
\text { [pont/mm }\end{array}$ & $\begin{array}{c}\text { Number of } \\
\text { points }\end{array}$ & $\begin{array}{c}\text { Number of } \\
\text { triangles }\end{array}$ & $\begin{array}{c}\text { Mean triangle } \\
\text { area }\left[\mathrm{mm}^{2}\right]\end{array}$ & $\begin{array}{c}\text { Mean triangle } \\
\text { side length }[\mathrm{mm}]\end{array}$ & Processing time & Expertise \\
\hline Artec & 5.1 & 110326 & 219574 & 0.10 & 0.51 & $\sim 30$ minutes & low \\
\hline TLS & 1.1 & 27118 & 52200 & 0.45 & 1.04 & $\sim 20$ minutes & high \\
\hline ScanArm & 3.9 & 83505 & 159326 & 0.14 & 0.57 & $\sim 5$ minutes & low \\
\hline Camera & 3.4 & 72416 & 141868 & 0.15 & 0.62 & $\sim 30$ minutes & medium \\
\hline Mobile & 3.7 & 78996 & 150935 & 0.14 & 0.60 & $\sim 30$ minutes & medium \\
\hline Sense & 1.0 & 20038 & 39672 & 0.51 & 1.13 & $\sim 5$ minutes & low \\
\hline
\end{tabular}

Table 2. Model parameters

Hernandez, 2015), but now the test aimed at only general cases. Both DSLR camera and mobile phone can be considered fully portable.

Terrestrial laser scanners (TLS) are used for scanning large, complex objects, e.g. buildings, structures and their environments; the manufacturers primarily recommend these instruments for civil engineering (building construction, excavation volumes, façade, structural deformations), forensics (crime scenes, accident details), industrial (product geometry, factories, process plants) and surveyings purposes. The scanner used in our test applies phase-based ranging, however, its accuracy is considered moderate in case of object scanning (see Table 1). The scanner is less portable compared to the other devices mentioned previously because of its many accessories.

The ScanArm scanner is even less portable, it should be deployed on a rigid, stable platform. According to manufacturer's claim the device can be used for reverse engineering, certification, part inspection, alignment, mould inspection, prototype part scanning projects in field of mechanical engineering. The size of the object to be scanned is limited by the 2.7-metres arm length. The scanner head emits a laser line and the coordinates of the surface points are determined based on the deformations of the line. Since this instrument must be connected to a computer or laptop for the operation, similar to the SLS scanners mentioned above, the already scanned parts of the object are visible during scanning, therefore the coverage can be continuously checked during measurement.

\section{MODELLING}

The endproducts of the mesaurements were point clouds or surface models (meshes); in case of point clouds there are many available modeling software environments (both open source and proprietary) capable of parameterizing and generating surface models.

Software provided by manufacturer is necessary for scanning with the Artec scanner and it is also used for primary data processing, which involves the refinement of the roughly aligned surface model. The method is very similar in case of Sense, except the model will be created automatically after measurement, there are no parameterization options, only the finished model can be modified. In case of TLS, first, the data must be downloaded from the scanner, then the point clouds captured from different scanner positions must be aligned and registered to each other using either the software provided by manufacturer or other solutions. Right after scanning with ScanArm, the mesh model can be exported. In case of the two photo based methods, point cloud and model could be created by reconstruction from multiple images technology using appropriate software, e.g. Agisoft Photoscan, Pix4D, Autodesk Remake and Visual SfM (Somogyi et al., 2017, Wikipedia, 2017, Jeon et al., 2017); for this study, Photoscan has been used for generating model from mobile pictures and Pix4D for the Camera model. Unscaled point clouds and models could be exported from these software, they must be scaled afterwards. Scaling ratio could be calculated if certain measures of the object are known; we used caliper to measure our test object.

Mesh models as measurement results can be seen in Figure 3, and they are compared to each other considering selected parameters in Table 2. Two rows could be highlighted in Table 2; in cases of TLS and Sense models, the point density is lower hereby less triangles could be observed. Artec model contains 220 thousand and ScanArm has 160 thousands while TLS includes 52 thousand and Sense consists only 40 thousand triangles.

As it can be seen in Table 3, the original Camera and Mobile model consist of millions of triangles (Camera - 3.3 million, Mobile - 4.7 million) which is many times bigger than the other models. These two models have been resampled for the comparison so the reduced versions contain around 150 thousand triangles. Of course, it has been investigated how big the difference is between the original and the resampled versions in both cases; the mean deviaton is less than $0.1 \mathrm{~mm}$. Table 3 contains the original point densities and model resolutions that have been calculated prior to resampling.

Manufacturers ensure software for their scanners (usually a single license is included in the scanner price) that are capable of generating models. Obviously, independent software must be used for creating models derived from picture based object reconstruction. As mentioned, many products are available on the market, besides free products, software can be chosen from wide price range ( 20-4000 EUR) (Wikipedia, 2017). Current trends tend to put even more emphasize on renting software for a certain period of time, and usually 15 or 30 day trial versions of the commercial software are also available. Two different trial products have been applied for the test; Camera model has been created by Pix4D and Photoscan has been used for the Mobile

\begin{tabular}{|l|c|c|c|c|c|}
\cline { 2 - 5 } \multicolumn{1}{c|}{} & $\begin{array}{c}\text { Original point density } \\
\text { [points } / \mathrm{mm}^{2} \text { ] }\end{array}$ & Number of points & Number of triangles & $\begin{array}{c}\text { Mean triangle } \\
\text { area [mm }{ }^{2} \text { ] }\end{array}$ & $\begin{array}{c}\text { Mean triangle side } \\
\text { length [mm] }\end{array}$ \\
\hline Camera & 85.4 & $\sim 1.9$ million & $\sim 3.3$ million & $<0.01$ & $<0.01$ \\
\hline Mobile & 110.3 & $\sim 2.4$ million & $\sim 4.7$ million & $<0.01$ & $<0.01$ \\
\hline
\end{tabular}

Table 3. Camera and Mobile models with original point densities 


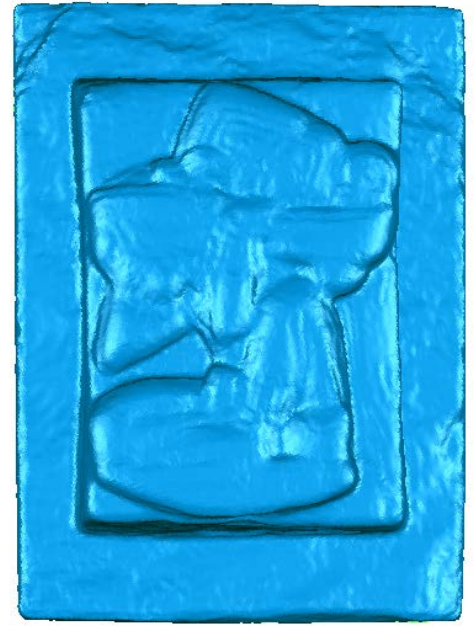

Artec

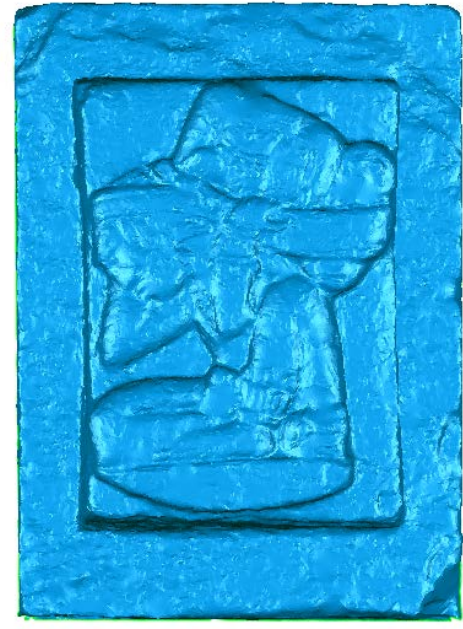

Camera

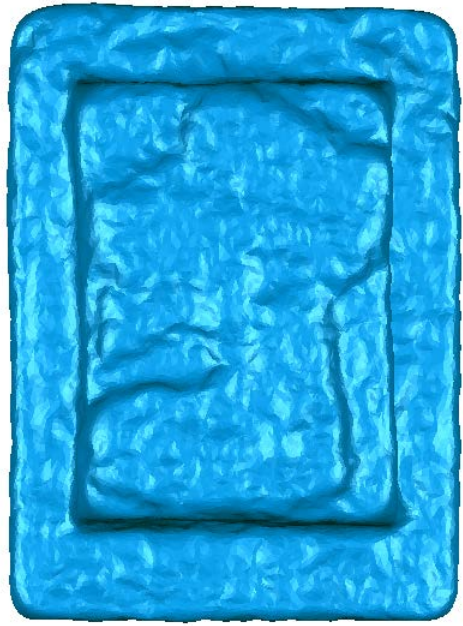

TLS

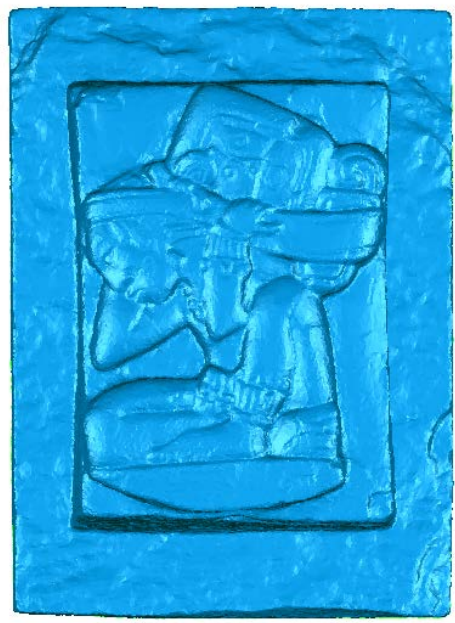

Mobile

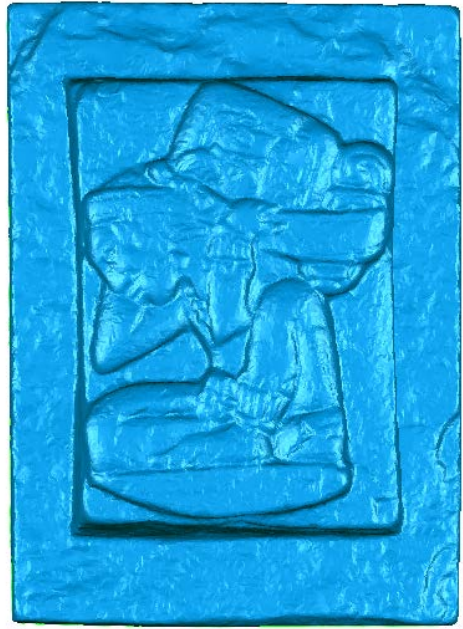

ScanArm

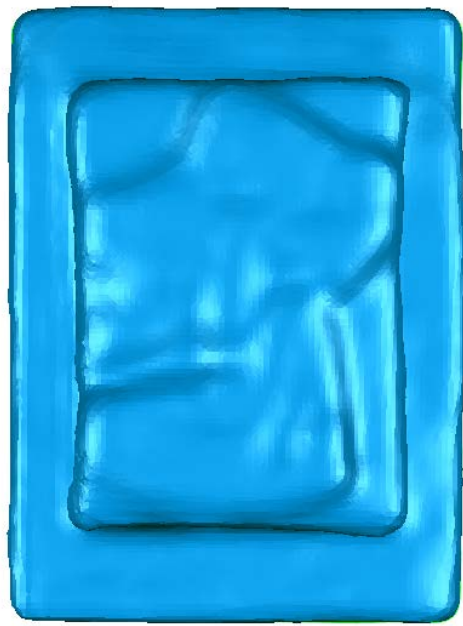

Sense

Figure 3. Triangulated surface models

model. A laptop with Intel Core i7-6700HQ 2.60 GHz processor, 8 GB memory, 128 GB SSD and 1 TB HDD has been used for data processing.

\section{COMPARISON}

Accuracy and resolution of the models have been evaluated; point density, number of triangles, mean triangle area and side length values can be seen in Table 2. These features give additional information for the comparison.

\subsection{Accuracy}

According to manufacturer claims and previous research results, the model derived from the ScanArm scanner was chosen as reference; it has about $0.1 \mathrm{~mm}$ measurement accuracy and in our particular case the model has 3.9 points $/ \mathrm{mm}^{2}$ average point density. The Artec, Sense, TLS, Camera and Mobile models have been compared to this reference. Deviation values that are visible in the tables, are displayed with 0.01 precision according to ScanArm's standard accuracy $(0.076 \mathrm{~mm})$.

As a first step, the 5 tested models have been aligned to the reference with ICP (Iterative Closest Points) algorithm without scaling (Low, 2004, Pomerleau et al., 2013). Therefore all 6 models are in the same coordinate system that enabled generating 3D deviation maps in each case by the built-in function of CloudCompare (Figure 4) (Cloudcompare, 2015). As it can be seen, Camera and Mobile models are similarly good, Artec model is a little bit weaker but TLS and Sense are much less accurate compared to the previous ones; the differences are located on much wider spectrum.

Besides the mean and deviation values of the differences, the volumes of models compared to the volume of reference model hold also useful information on global quality, the values are shown in Table 4. It can be seen that the numerical values give less information than the visual information in this case; the differences between the deviation maps are not reflected by the numbers. 


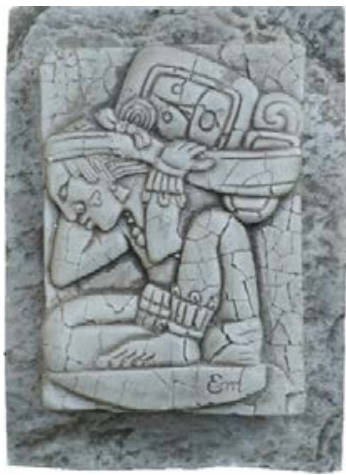

Photo

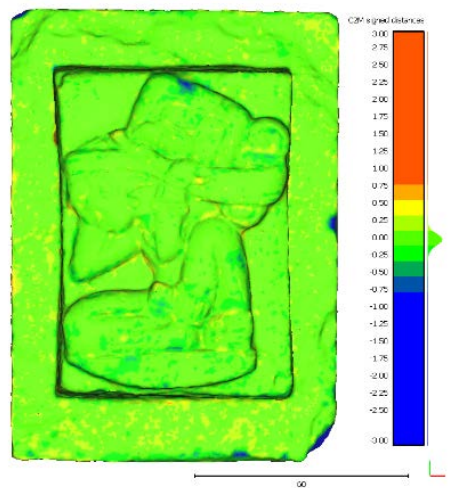

Camera

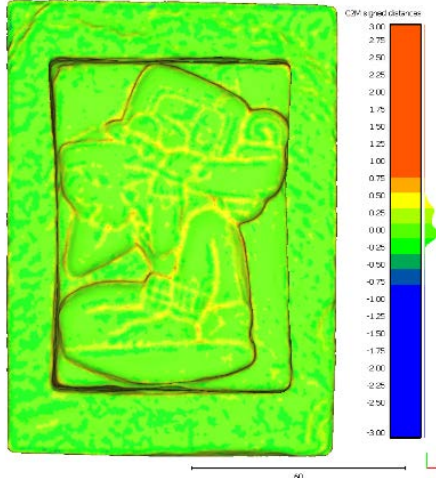

Artec

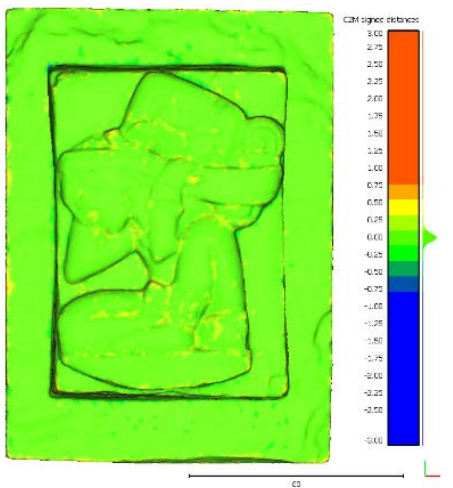

Mobile

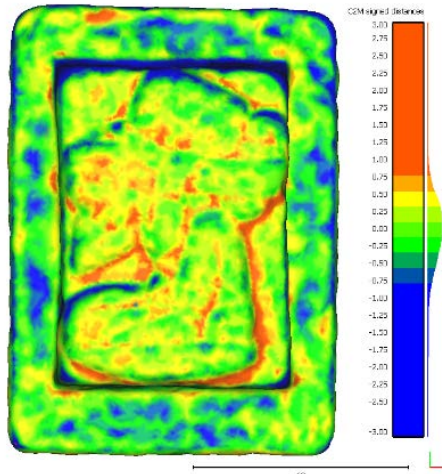

TLS

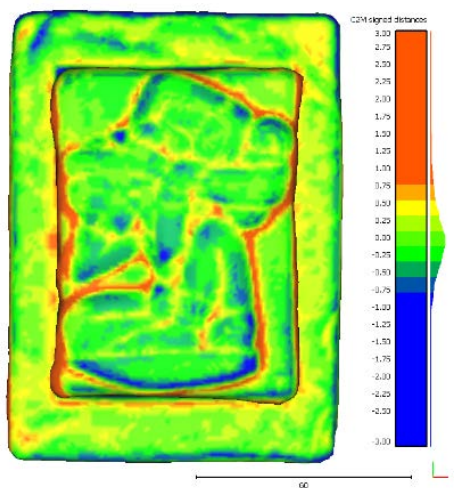

Sense

Figure 4. Deviation maps of the surface models compared to the reference

Since the documentation of the 3D deviation map generating function is not available in case of the applied software, it operates as a black box; the users do not know exactly which algorithms and parameters are used. Therefore, the derived differences also have been analysed in an other way, by using 2D cross-sections. Since each model has been transformed into the same coordinate-system, $1 \mathrm{~mm}$ thick cross-sections have been generated $1 \mathrm{~cm}$ from each other in 3 orthogonal directions (near parallel to the sides of sculpture). In direction $X$ (i.e. parallel to the shorter side in top view) 13, in direction $Y$ (i.e. parallel to the longer) 9 and in direction Z 3 cross-sections have been invesitgated (see Figure 5).

Differences from the reference model have been calculated in each points of each cross-sections then basic statistics have been derived, results can be seen in Table 5 .

In each cross-section (see Figure 5) the distances between the reference and the particular points have been calculated.

\begin{tabular}{|l|c|c|c|}
\cline { 2 - 4 } \multicolumn{1}{c|}{} & $\begin{array}{c}\text { Differences } \\
{[\mathrm{mm}]}\end{array}$ & $\begin{array}{c}\text { Volume } \\
{\left[\mathrm{mm}^{3}\right]}\end{array}$ & $\begin{array}{c}\text { Difference of } \\
\text { volumes [\%] }\end{array}$ \\
\hline ScanArm & - & 182794 & - \\
\hline Artec & $0.20 \pm 1.14$ & 184877 & 1.14 \\
\hline Camera & $-0.20 \pm 1.03$ & 182039 & -0.41 \\
\hline Mobile & $-0.14 \pm 1.27$ & 182954 & 0.09 \\
\hline Sense & $-0.11 \pm 0.98$ & 183551 & 0.41 \\
\hline TLS & $-1.02 \pm 2.77$ & 178709 & -2.23 \\
\hline
\end{tabular}

Table 4. Deviation values of the models compared to the reference
Besides mean value, standard deviations belonging to each crosssection have also been derived.

Similar to what the deviation maps show, it can be seen from the values that Artec, Camera and Mobile models has one order of magnitude better accuracy compared to that of Sense and TLS.

Contours of the particular models derived from the cross-sections are illustrated together in Figure 6. While red, purple and green lines (Artec, Camera, and Mobile) nicely follow the reference (black), in cases of the blue and orange lines (TLS and Sense) the extent and frequency of deviation is remarkably higher. The biggest differences in the $\mathrm{X}-\mathrm{Y}$ cross sections can be observed at the top of the sculpture (where the fine details are found) and at the fine pattern parts. Note that lines both under and above the reference can be found. The same kind of results can be observed in the other cross-sections. Such 2D analysis reasonably supplement the 3D deviation maps by showing the characteristics of the deviations.

\begin{tabular}{|l|c|c|c|}
\cline { 2 - 4 } \multicolumn{1}{c|}{} & Meanx $[\mathrm{mm}]$ & Meany $[\mathrm{mm}]$ & Meanz $[\mathrm{mm}]$ \\
\hline Artec & $0.17 \pm 0.16$ & $0.15 \pm 0.14$ & $0.27 \pm 0.11$ \\
\hline Camera & $0.10 \pm 0.12$ & $0.10 \pm 0.11$ & $0.17 \pm 0.24$ \\
\hline Mobile & $0.09 \pm 0.11$ & $0.08 \pm 0.07$ & $0.08 \pm 0.06$ \\
\hline Sense & $0.39 \pm 0.38$ & $0.37 \pm 0.32$ & $0.70 \pm 0.48$ \\
\hline TLS & $0.39 \pm 0.32$ & $0.47 \pm 0.47$ & $0.65 \pm 0.66$ \\
\hline
\end{tabular}

Table 5. Statistics of differences deriving from cross-sections 


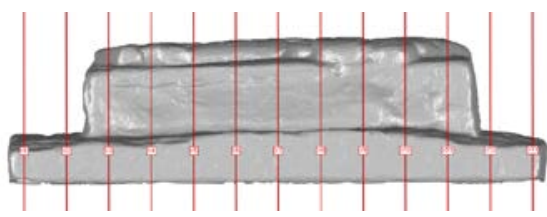

Direction X

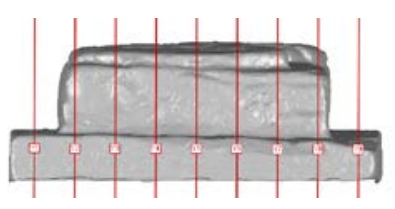

Direction Y

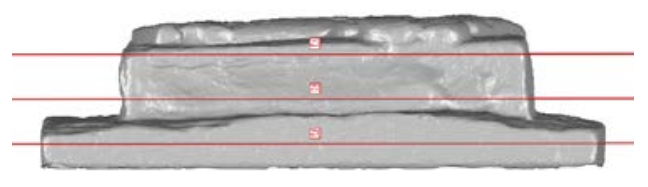

Direction Z

Figure 5. Location of cross-sections in three directions

\subsection{Resolution}

The resolution largely depends on the point density that is significantly different at the investigated models; TLS and Sense are much less dense than the others (see Table 2). Only the middle part of the sculpture has been involved into the resolution analysis; this part has been cropped out from each model. It has been investigated how well the fine drawings, carvings look on the models. The own-developed local minimum maps have been generated with the same properties in case of each model and they nicely visualize the potential level of detail in Figure 7 and 9. These local minimum maps highlight the deep points, i.e. those that are mostly surrounded by higher points.

The figures would be able to get quantified in several ways, e.g. ratio of the deep points compared to all points; but it would include also false deep-points which may originate from measurement inaccuracy. The obtained contur maps ensure a new method of comparing the level of detail for each models; future research aims at developing numerical analysis of these models.

As it was previously mentioned, the Camera and Mobile models has high point density; Figure 8 shows that the local minimum maps derived from original point density appear more clearly on such dense point clouds but false deep points have also been identified, may have caused by noise.

\section{EVALUATION OF RESULTS}

The result of the accuracy test met with the prior expectations; Artec and ScanArm modell are much more accurate than Faro and Sense model and the accuracy of models generated from Camera and Mobile images are remarkable.

As previously mentioned, at the Sense measurement setting the parameters was not an option, therefore strong smoothing and resampling cannot be avoided, the fine drawing cannot be seen. In case of TLS model, noise can be observed because of the ranging accuracy of the scanner; many non-deep points have been marked as deep ones.

While Artec scanner project a pattern onto the object, ScanArm scanner head emits a laser line during operation; both of them detect the reflected pattern/line then 3D coordinates are calculated based on the detected pattern deformation. Due to geometric constrains the instrument can hardly or cannot measure into certain depth and narrow places since the emitting and detecting sensors cannot be any close to each other. The measurement capability limits of the instrument and this effect (i.e. unable to detect the very deep points) seems in case of Artec model at cross-sections, 3D deviation maps and local minimum map, too.

The Camera and Mobile models can be produced by many different software, however, there can be significant differences among the models from diferrent sources. The test resulted that the Camera

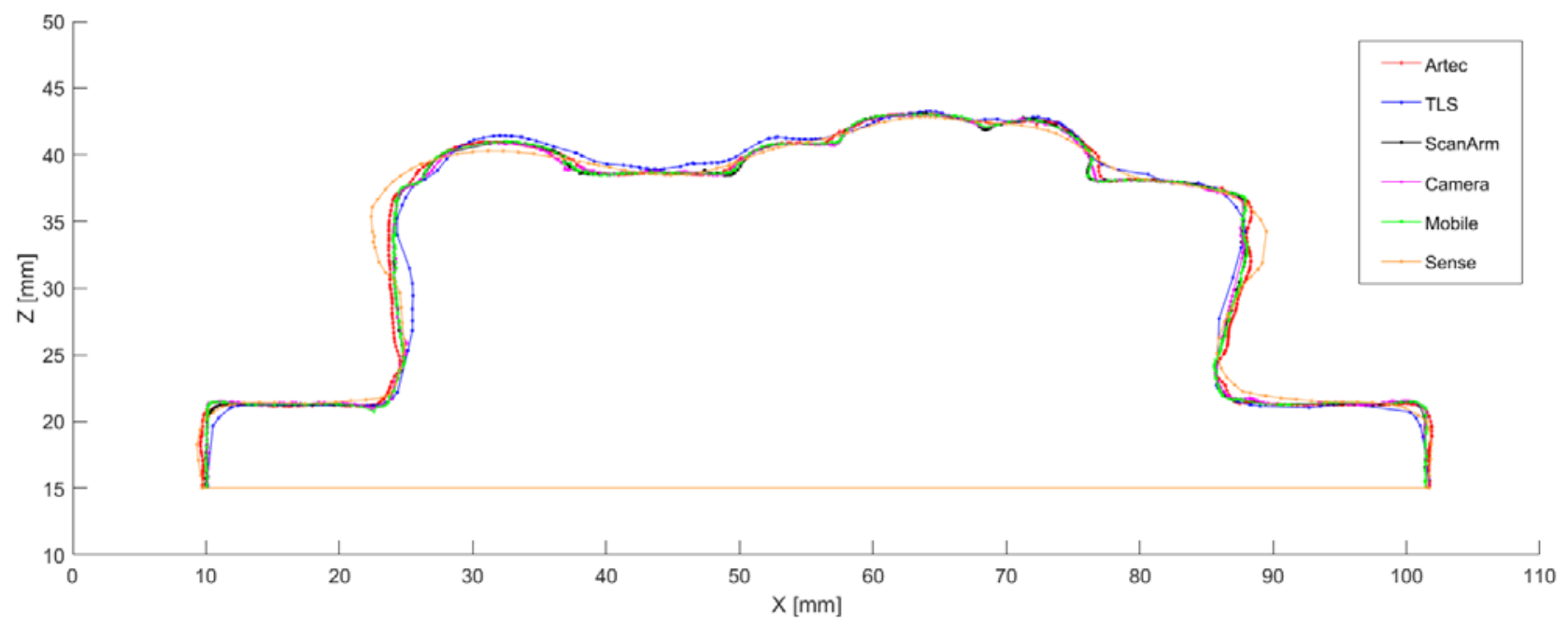

Figure 6. Model contours in a cross-section 
The International Archives of the Photogrammetry, Remote Sensing and Spatial Information Sciences, Volume XLII-2/W8, 2017 5th International Workshop LowCost 3D - Sensors, Algorithms, Applications, 28-29 November 2017, Hamburg, Germany
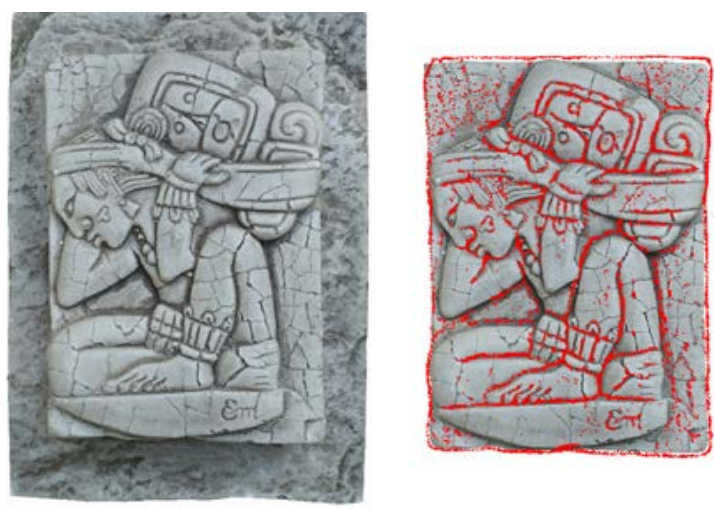

Figure 7. Photo of the test object (left) and the deep points of ScanArm model projected onto the photo (right)
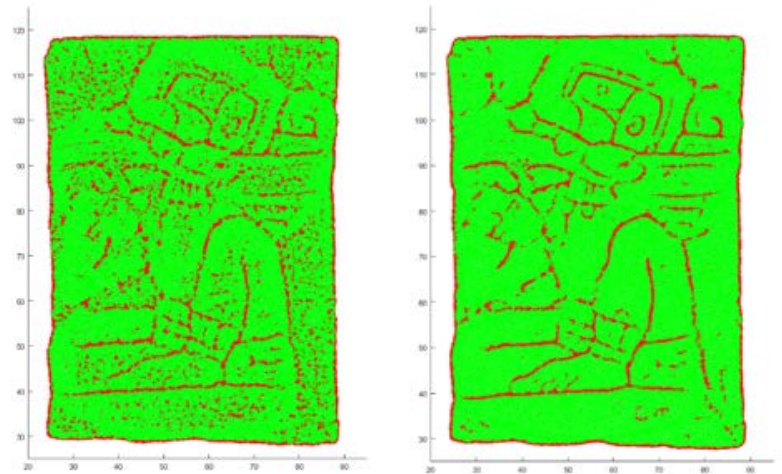

Figure 8. Local minimum maps (original point density); Camera model (left), Mobile model (right)

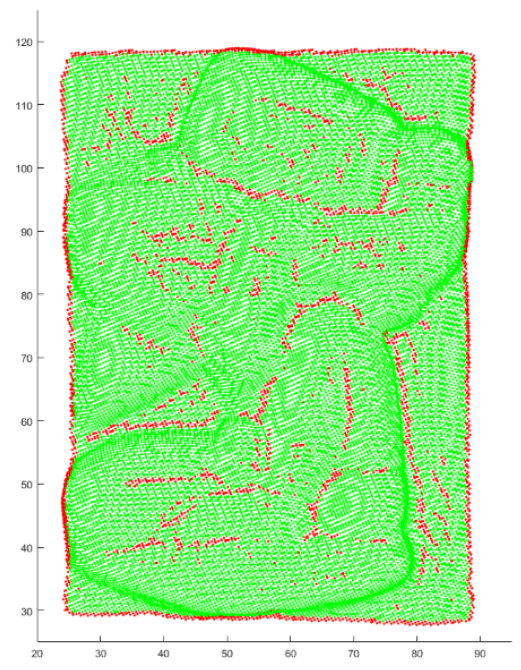

Artec

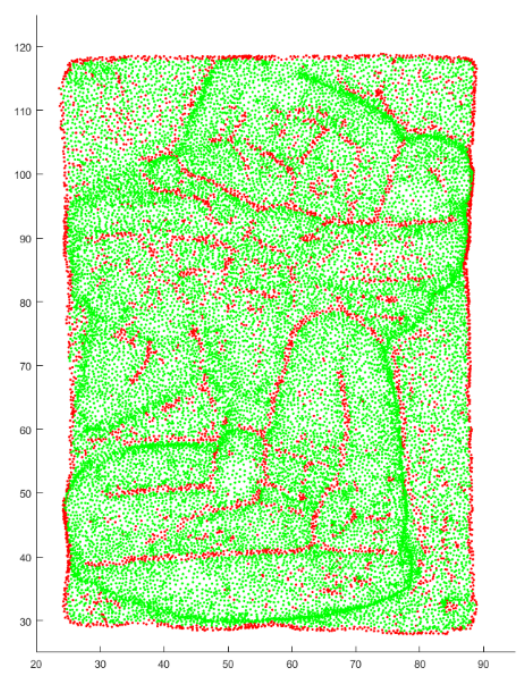

Camera

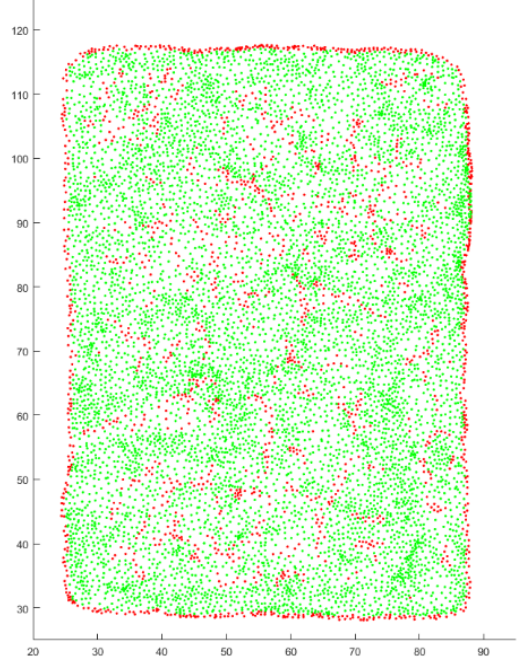

TLS

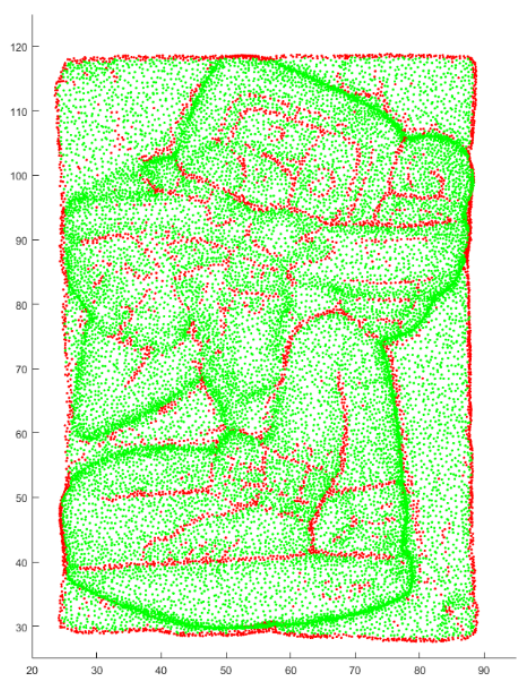

Mobile

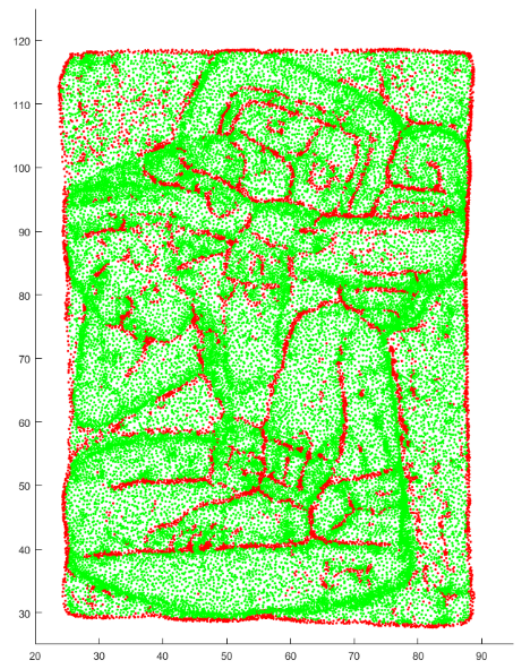

ScanArm

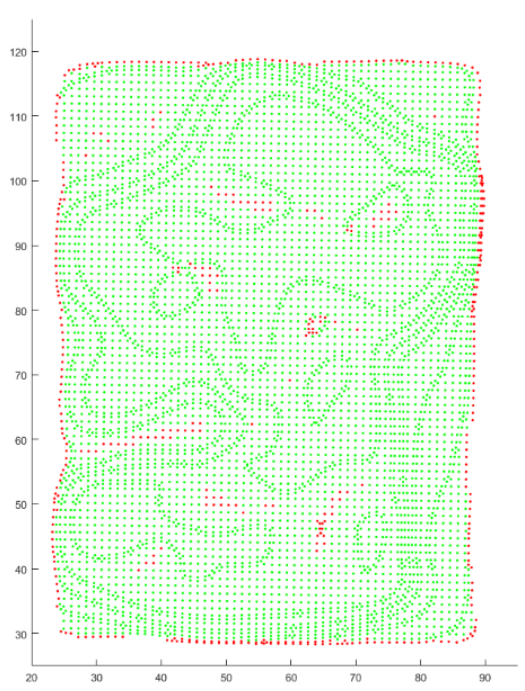

Sense

Figure 9. Local minimum maps; red - deep points, green - non-deep points 
and Mobile model has similar accuracy but resolution of the Mobile model is a bit better than that of the Camera model. It does not mean that mobile images generally enable better models; the applied software has a great impact on the model attributes. There are studies (Somogyi et al., 2017, Barazzetti et al., 2010, Bianconi, et al., 2017) about the effect of combination of devices and software, this is not detailed in the current study; just two available $3 \mathrm{D}$ dense models have been tested. The conclusion can be deducted that models generated from images (camera and mobile) by pixelbased 3D reconstruction technology have almost the same accuracy and resolution and they can compete with the ScanArm model in many potential applications.

An other important viewpoint by selecting the measurement device whether texture is necessary or not. Coloured point clouds or models can be generated from all the insturments involved into the test except the ScanArm scanner. It may occur that there is no sufficient ambient light during measurement; image based solutions, Artec and Sense scanners cannot be used in the dark but it does not cause any problems for the TLS and ScanArm scanner. Other obstructive circumstances like reflective surfaces could emerge in certain measurements, which do not appear properly in the photos and also influence the laser based solutions.

It can be observed on the local minimum maps that point density is an important influential factor, since the drawing/pattern barely can be seen by low density (see Faro and Sense modell) while Camera and Mobile models from original point densities are very detailed. High point density itself is not enogh for high resolution model, e.g. Artec provides high point densitiy, but since it cannot catch the very deep points due to its geometric constrains (see above), a smoothening effect can be observed; surface will be generated above the undetected deep points.

\section{CONCLUSION}

Our goal was to find the best technology to survey a small, detailed object by thorough investigation of selected parameters. From the results it seems that TLS is not suitable for measuring such smallsize objects accurately and in high resolution. It can be also stated that although the Sense scanner is suitable for measuring and modeling small objects, it is not recommended for fine and precise modelling. Its reasonable price (i.e. some smart phones are even more expensive than this device) makes it competitive at works where high level of detail is not important. Artec scanner produces moderately detailed but very accurate model of smaller objects but the device is almost as expensive as a TLS. The ScanArm scanner resulted the best model, therefore it was used as reference in the analysis. In terms of price it is not considered an affordable solution at all, it is the most expensive among the others, but if this level of precision and resolution are needed, the price has to be payed. The pixel based 3D reconstruction solutions can be a reasonable choice because detailed, accurate model can be produced by a device which is in almost everyone's pocket; only the software charges are needed to be paid if not a free solution has been chosen. However, finding the appropriate software is not a straightforward task and it greatly influences the result. The measuring time is similar in each case and there can be no outstanding value found at analyzing the processing times either. Selecting the best instrument to a particular survey depends on the weight of paramters to be considered. Our investigations show that even low-cost sensors should be considered in such projects.

As further research perspective, comparative study is planned to do for larger-size objects. Motivated by the remarkable results of the mobile phone, investigation is planned involving several, different kinds of mobile phones using the same 3D reconstruction software.

\section{REFERENCES}

Barazzetti, L., Remondino, F. and Scaioni, M., 2010. Automation in 3D reconstruction: results on different kinds of close-range blocks. The International Archives of the Photogrammetry, Remote Sensing and Spatial Information Sciences, Vol. XXXVIII, Part 5, pp. 56-61.

Bianconi, F., Catalucci, S., Filippucci, M., Marsili, R., Moretti, M., Rossi, G. and Speranzini, E., 2017. Comparison between two non-contact techniques for art digitalization, Journal of Physics: Gnference Series, 882(1).

CloudCompare, 2015. “Cloud-to-Mesh Distance”, http://www.cloudcompare.org/doc/wiki/index.php?title=Cloud-toMesh_Distance (18 Oct. 2017).

Fofi, D., Sliwa, T. and Voisin, Y., 2004. A comparative survey on invisible structured light. Proceedings of SPIE. Vol. 5303, pp. 90-98.

Furukawa, Y. and Hernandez, C., 2015. Multi-ViewStereo: A Tutorial. Foundations and Trends ${ }^{\circledR}$ in Computer Graphics and Vision, Vol. 9, No. 1-2, pp. 1-148.

Jeon, E.-I., Yu, S.-J., Seok, H.-W., Kang, S.-J., Lee, K.-Y. and Kwon, O.-S., 2017. Comparative evaluation of commercial softwares in UAV imagery for cultural heritage recording: case study for traditional building in South Korea. In: Spatial Information Research, Springer, Singapore, pp. 1-12.

Low, K.-L., 2004. Linear least-squares optimization for point-toplane ICP surface registration. Technical Report TR04-004, Department of Computer Science, University of North Carolina at Chapel Hill, NC, USA.

Moons, T., Vergauwen, M. and Gool, L.V., 2008. 3D Reconstruction from Multiple Images. Now Publishers, United States, p. 129.

Pomerleau, F., Colas, F., Siegwart, R. and Magnenat, S., 2013. Comparing ICP variants on real-world data sets Opensource library and experimental protocol. Autonomous Robots, Springer Verlag, 34(3), pp. 133-148.

Somogyi, A., Lovas, T. and Barsi, A., 2017. Comparison of spatial reconstruction software packages using DSLR images. Pollack Periodica, 12(2), pp. 17-27.

Wikipedia, 2017. "Comparison of photogrammetry software”, https://en.wikipedia.org/wiki/Comparison_of_photogrammetry_s oftware (18 Oct. 2017). 